\title{
Lymphoid Follicle
}

National Cancer Institute

\section{Source}

National Cancer Institute. Lymphoid Follicle. NCI Thesaurus. Code C33040.

Nodular structures composed of lymphocytes. In the absence of immune stimulation,

they ae called primary follicles and are composed of small B-lymphocytes. With antigenic stimulation the B-lymphocytes replicate and differentiate, to give rise to secondary follicles which are composed of germinal centers and are surrounded by mantle zones. 